\title{
Soundjata ou l'epopée mandingue à la lumiere de La structure du mythe du héros ou le désir d'etre Dieu de Philippe Sellier
}

\author{
Dame KANE \\ Université Cheikh Anta DIOP, Sénégal \\ Reçu le 27 avril 2018 | Évalué le 29 avril 2018 | Accepté le 29 juin 2018
}

\begin{abstract}
RÉSUMÉ. L'intrusion de l'oralité dans les champs de recherche universitaire d'Afrique noire dans les années 1960, a mis au grand jour la richesse des récits épiques maliens. Plusieurs possibilités se présentent dans leur étude notamment l'analyse de leurs liens avec l'épopée occidentale. Ce qui pourrait se faire en nous appuyant sur l'approche de Sellier (1970) suivant une démarche comparative. Soundjata, figure historique fondatrice de l'empire du Mali et initiatrice de la charte du Manden établie à kouroukan Fouga avec ses alliés de Kirina ayant triomphé du Roi forgeron Soumaoro Kanté à la bataille de Kirina en1235, devient, de ce fait, un personnage épique. Son épopée chantée par les griots, autrefois détenteurs de l'histoire, sera reprise par plusieurs auteurs comme Niane (1960) dans Soundjata ou l'épopée mandingue qui exalte les exploits de ce Roi-lion qui continue de marquer la mémoire des africains notamment de son peuple. Dans cet article, il est question, donc, de voir si cette œuvre de Niane prend en considération les différentes étapes du modèle héroïque classique tel qu'il est présenté par Sellier (1970) dans La structure du mythe du héros ou le désir d'être Dieu.
\end{abstract}

Mots-clés : épopée, héros, manding, négro-africain, Sellier, Soundjata

\begin{abstract}
The intrusion of orality into the academic research fields of black Africa in the 1960s brought to light the richness of Malian epic narratives. Several possibilities exist in their study, including the analysis of their links with the Western epic. This could be done by using the Sellier approach (1970) using a comparative approach. Soundjata, founding historical figure of the Malian empire and initiator of the Manden charter established at Kurukan Fouga with his Kirina allies having triumphed over the blacksmith king Soumaoro Kanté at the Battle of Kirina in 1235, becomes, therefore, an epic character. His epic sung by the griots, formerly holders of the story, will be taken up by several authors like Niane (1960) in Soundjata or the Mandingo epic that extols the exploits of this Lion King who continues to mark the memory of Africans including his people. In this article, it is a question, therefore, to see if this work of Niane takes into consideration the different stages of the classic heroic model as presented by Sellier (1970) in The structure of the myth of the hero or the desire of to be God.
\end{abstract}

Keywords: épic, héro, manding, négro-african, Sellier, Soundjata

auteur correspondant : Dame_4@hotmail.com

Pour citer cet article (Style APA) : Kane, D. (2018). Soundjata ou l'épopée mandingue à la lumière de la structure du mythe du héros ou le désir d'être dieu de Philippe Sellier. Francisola: Revue Indonésienne de la langue et la littérature françaises, 3(1), 88-94. doi: http://dx.doi.org/10.17509/francisola.v3i1.11894 


\section{INTRODUCTION}

La littérature négro-africaine était, jusqu'au début du XXème siècle, dominée par les genres oraux tel que le mythe, la légende mais surtout l'épopée. Quelle soit royale s'intéressant aux héros des dynasties ou religieuse relatant le parcours d'un dignitaire religieux ou corporative mettant au devant de la scène un haut personnage qui s'est merveilleusement illustré dans sa corporation ou mythologique clanique dans laquelle l'influence du clan fait émerger des héros marginaux, l'épopée a su se diversifier et s'enrichir au cours des siècles. De l'Atlantique à la corne de l'Afrique, les récits épiques forment ce que Dieng (1997) appelle une épic belt et mettent en exergue des figures historiques majeures qui ont marqué leurs communautés. C'est le cas de Soundjata Keita, empereur du Mali au XIIIeme siècle et dont les exploits sont toujours exaltés par les griots modernes. Cissé (1988) de même que Camara (1978), mais surtout Niane (1960), ont repris la trajectoire de ce mythique chef malien. Les définitions qui sont données de l'épopée foisonnent mais semblent toutes complémentaires et ont en commun l'existence d'un personnage héroïque qui domine toute la trame. Mais celle donnée par Nicole Revel, docteur ès lettres et sciences humaines, directeur de recherche au C.N.R.S, semble être la plus pertinente :

Proche du mythe, l'épopée chante l'histoire d'une tradition, un complexe de représentations sociales, politiques, religieuses, un code moral, une esthétique. À travers le récit des épreuves et des hauts faits d'un héros ou d'une héroïne, elle met en lumière un monde total, une réalité vivante, un savoir sur le monde. (Revel et al, 1966).

\section{Pour Zumthor (1982), l'épopée est un}

Récit d'action concentrant en celle-ci ses effets de sens; économe d'ornements annexes, l'épopée met en scène l'agressivité virile au service de quelque grande entreprise. Fondamentalement elle narre un combat et dégage parmi ses protagonistes une figure hors $\mathrm{du}$ commun qui, pour ne pas sortir toujours vainqueur de l'épreuve, n'en suscite pas moins l'admiration.

Le héros épique suivrait ainsi un cheminement particulier qui le ferait rayonner dans la durée. D'Héraclès aux figures extraordinaires des temps modernes, le modèle héroïque semble obéir à une logique cardinale qui devient un véritable instrument d'analyse du genre épique. Cette structure permanente est décrite, pour la première fois, par Rank (1909) dans Le Mythe de la naissance du héros. Mais c'est avec Sellier (1970) que l'on a en une version plus limpide.

Cependant toutes ces études n'ont pour références que les épopées classiques occidentales et n'abordent nullement les textes épiques négro-africains qui, pourtant, sont d'une grande richesse. Il serait ainsi légitime de voir si l'itinéraire de Soundjata, figure emblématique malienne, tel qu'elle est exposée dans cette œuvre, est conforme à la structure du mythe héroïque de Sellier, de la naissance du héros à son immortalité qui est une forme de renaissance succédant à la mort physique de ce dernier. Le choix de Soundjata ou l'épopée mandingue n'est pas fortuit. Il trouve son explication au fait que: «nous devons ainsi l'établissement du premier texte épique à l'historien Djibril TamsirNiane» (Dieng, 1997). Avant, les récits se limitaient exclusivement à leur double dimension, à la fois historique et orale, l'écriture étant inexistante. La parole du griot Mamadou Kouyaté, les premiers rois du Manding, la femme-buffle, l'enfant-lion, l'enfance, le réveil du lion, l'exil, Soumaoro Kanté, le roisorcier, Histoire, les feuilles de baobab, le retour, le nom des héros, Nana, Tribanet Balla Fasséké, Krina, l'Empire, KouroukanFougan ou le partage du monde, Niani, le Manding éternel, sont les grandes lignes de cette œuvre mettant à jour l'une des plus illustres épopées d'Afrique subsaharienne.

\section{MÉTHODE}

L'épopée mandingue de Niane reste largement fidèle à l'histoire relatée par les kouyaté, griots du Mali, avec une forte présence des marques de l'oralité qui vont des proverbes aux interactions verbales en passant par les interjections et une 
présentation particulière des structures du récit. Son héros a évolué dans un cadre historique marqué par une forte rivalité entre les chasseurs groupe socio-professionnel de Soundjata Keita et les forgerons dont la figure la plus illustre demeure Soumaoro Kanté le Roi-sorcier qui régnait à Sosso et qui était craint par tout son peuple du fait notamment de ses multiples exactions et de ses pouvoirs magiques. La structure hérö̈que de Sellier est l'outil qui va nous permettre de cerner la dimension mythique du héros mandingue. L'approche que nous avons choisie s'appuie sur des extraits épiques au prisme du mécanisme Sellierienne. Ce qui exige des relevés de passages illustratifs de notre corpus combinés à la structure d'appuie. Ces derniers seront repérés, transcrits, analysés en fonction de l'orientation de cette étude qui nous donnera la possibilité de comparer les modèles épiques occidental et négro-africain. Ce dernier reste jusqu'ici très peu exploré comme le souligne déjà Dumezil (1949) à travers L'Héritage indo-européen à Rome repris par Dieng (1997) dans Les épopées d'Afrique noire:

Tel est...l'état des recherches. Etat, comme il a déjà été dit, tout provisoire. Des chantiers sont ouverts, des formes archaïques ont apparu, ont été hâtivement dégagées inventoriée, des cheminements mènent de l'une à l'autre. Mais des vestiges considérables dorment peut-être encore en des points où nous ne songeons pas à porter la pioche; peutêtre tout ce que nous voyons est-il appelé à changer de sens, à prendre place dans un ensemble plus vaste différemment organisé. Du moins en voyons-nous assez pour être assuré qu'il ne s'agit ni d'un mirage fugitif ni d'une construction gratuite (P.11)

En nous efforçant ainsi de confondre l'épopée mandingue typiquement négroafricain et la structure du mythe hérö̈que occidentale classique nous pourrons nous rendre compte tant de leurs similitudes que de leurs dissemblances. Les huit étapes constituantes du modèle de Sellier (1970) qui sont des caractéristiques de l'épopée depuis l'antiquité, se présentent ainsi :
- Les présages: le héros nait de parents illustres de nature divine ou appartenant souvent à la haute société

- L'exposition: des prédictions défavorables font qu'il est exposé au danger ou séparé de ses parents.

- L'occultation: sa difficile naissance et l'atmosphère périlleuse dans laquelle il évolue font qu'il est presque invisible et mène une existence cachée.

- La reconnaissance par le signe :mis à l'épreuve le héros signe la mort du personnage anonyme qu'il était et renait sous visage nouveau il passe ainsi d'une vie cachée à la célébrité.

- L'épiphanie héroïque: les qualités exceptionnelles du héros éclosent au grand jour. Il relève les défis les plus insurmontables.

- Le sauveur: Le héros sauve son peuple généralement des exactions d'un tyran.

- L'initié : Son ascendance sur les hommes fait de lui un proche de Dieu.

- L'apothéose: c'est la réincarnation, l'accession à l'immortalité par l'exaltation des exploits du héros après sa disparition physique.

Une étude de cette structure octogonale montre qu'elle peut être condensée en quatre phases qui la synthétisent de manière exhaustive sans biaiser son essence. C'est sur cette base que se fera notre analyse. Il s'agit de la naissance du héros épique, son enfance, son affirmation en tant que héros et de son triomphe aussi bien des multiples épreuves qui ont jalonnés son parcours que de la mort.

Etudes africaines, revue littéraire s'intéressant surtout aux questions qui interpellent les littératures négro-africaines orale et écrite d'expression française ou traduite de même que le laboratoire de l'oralité rattaché à l'école doctorale ARCIV (Art Culture et Civilisation ) de l'université Cheikh Anta Diop ont permis la production de texte qui reviennent largement sur l'épopée sous les avis éclairés de plusieurs spécialistes de ce domaine notamment les Professeurs feu Bassirou Dieng, Amadou Ly, Samba Dieng. Ce dernier s'est largement inspiré de Sellier dans Sur les traces d'Elhadj Omar et la structure du mythe héroïque de 
Sellier (2009). Sa pertinence réside en ce qu'elle présente un canevas qui prend en charge les épopées les plus illustres du monde occidental. Notre démarche se veut analytique en recensant pour chaque phase de cette structure permanente des illustrations référées qui seront soutenues par des commentaires explicites qui nous permettront d'arriver aux résultats escomptés. C'est-à-dire de mettre la lumière sur l'insertion de l'épopée négro-africaine en général et en particulier malienne dans le modèle épique classique.

\section{RÉSULTATS ET DISCUSSION}

L'histoire est indissociable de l'épopée car elle en constitue la substance à laquelle s'est ajouté le merveilleux. Si l'on tient compte des faits historiques relatés dans L'empire du Mali de Ly-Tall (1977), on constate que c'est l'association, par le sang et le jeu des alliances, de deux royaumes, le Sosso et le Manden, après le déclin du wagadu-Ghana, que l'empire du Mali va se construire et connaitre une grande émergence au XIIIème siècle avec une organisation socio-politique particulière qui le fera brillé pendant des siècles. Occupant la totalité de l'espace malien actuel et une bonne partie du Sénégal, de la Cote d'ivoire et de la Guinée, Cet empire avait la réputation d'être un cadre cosmopolite où l'on rencontrait des ethnies et des peuples d'une grande diversité: les Masufa ( nomades et éleveurs), les Berbères (grands commerçants), les Wolofs, les Bambaras et les Mandenka qualifiés de peuples de la savane, les Sahéliens essentiellement des nomades du Soudan, les Sonikés et les toucouleurs, le peuple Dogon cantonné sur les falaises de Bandiagara, une région difficile à accéder. Ce qui en faisait une communauté loin de l'emprise des Rois mandingues. C'est donc ce vaste et riche empire du moyen-âge ouest africain que le héros Soundjata a réussi à former et à diriger jusqu'à sa disparition.

Ce personnage héroïque est issu d'une famille royale. Son père Naré Maghan Konaté fut le Roi du Manding ayant pour capitale Niani une localité de l'Afrique occidentale. Surnommée la femme-buffle, investie de pouvoirs magiques qui la singularisent des autres épouses de Maghan, Sogolon Kondé, mère du héros de Niane, va jouer un rôle déterminant dans l'ascension de ce dernier. Leur union s'est faite suite au pacte entre Do-Kamissa sœur ainée du Roi de DÔ d'une part et d'autre part Dan Massa Woulani et Dan Massa Woulamba, deux chasseurs qui voulaient mettre fin au pouvoir de Do-Kamissa de se métamorphoser en buffle.

Au delà de la dimension hors du commun de ses parents, Soundjata a connu une naissance accompagnée de circonstances inhabituelles :

Soudain le Ciel s'assombrit, de gros nuages venus de l'est cachèrent le soleil ; pourtant on était en saison sèche; ; quelques grosses gouttes de pluie se mirent à Tomber tandis qu'un vent effroyable s'élevait ... C'est à ce

Moment que sortit une matrone de la case de Sogolon; elle courut vers le vestibule et annonça à NaréMaghan qu'il était père d'un garçon... les Coups précipités du tambour royal annoncèrent au Manding la naissance d'un fils. (Niane, 1960, p.34)

Les signes de la nature montraient que ce nouveau-né est différent des autres. Ce qui laissait penser la venue au monde d'un sauveur, d'un messie ou simplement d'un grand homme qui va marquer son époque et l'histoire de son peuple. Les présages de Sellier se confirment ainsi dans la naissance de Soundjata qui connaitra aussi une enfance tumultueuse.

Mais cette période marquée de signes fortes ne sera pas d'une grande utilité pour le devenir du héros qui, Selon Sellier (1970), va meneralors une existence obscure, bien différente de celle à laquelle sa naissance eut du le faire céder. C'est la période d'une vie cachée,d'une mort apparente.

Le héros manding va surprendre son entourage notamment sa mère $\mathrm{du}$ fait qu'il resta jusqu'à dix ans sans marcher ni parler. Cette dernière sera ainsi le quolibet de la cours royale notamment de la première épouse du RoiSassoumaBereté qui tente de la ridiculiser en permanence favorisant son propre fils. La perspective de la future 
succession du Roi ne rend pas la vie facile à Soundjata potentiel héritier de Maghan. Son handicap lui fera subir de multiples affronts :

La méchante reine-mère laissait la voie libre à tous les curieux qui voulaient voir l'enfant qui, à sept ans, se traînait encore par terre; presque tous les habitants de Niani défilèrent dans le palais; la pauvre Sogolon pleurait de se voir ainsi livrée à la risée publique. ... Sogolon ne trouvait un peu de consolation que dans l'amour de sa première fille, Kolonkan; elle avait quatre ans et marchait... Elle semblait comprendre toutes les misères; de sa mère (p.42)

Cette période difficile dans la vie du héros que Sellier appelle l'occultation, peut être interpréter comme un moment d'initiation d'apprentissage qui va fortement contribuer au renforcement de la personnalité du héros. La souffrance permet dans un certain sens de se fortifier de s'élever au dessus de la facilité en poussant toujours plus loin les limites de l'individu. Cette souffrance constructrice de grandeur et facteur de connaissance de soi et d'autrui, est bien connu par Musset, écrivain romantique victime et témoin de maux multiples liés à l'existence. Aussi dira-t-il dans Nuit d'octobre (1837) : "L'homme est un apprenti la douleur est son maitre nul ne peut connaitre la vie 'sil n'a pas souffert. » Le héros se sent ainsi investi d'une mission celle de montrer aux autres qu'il est capable de grandes choses malgré les écueils de la vie présente.

Chez Soundjata, le rôle de la mère est incontournable dans la construction de la figure du héros. Elle sera au début et à la fin de l'itinéraire de ce personnage épique. C'est son attitude exemplaire qui poussera son enfant à surmonter, de manière merveilleuse, certaines offenses. Insultée par sa tante Sassouma, Soundjata n'en pouvant plus d'être le témoin et la victime passive de tant de méchanceté se révolta :

-Mère, qu'y a-t-il ?

-Tais-toi, rien ne pourra Jamais me laver de cet affront.

- Maisquoidonc?

-Sassouma vient de m'humilier pour une histoire de feuille de baobab.A ton âge son fils à elle marchait et apportait à sa mère des feuilles de baobab.

- Console-toi,mère,

Console-toi!

-Non,C'esttrop,jenepuis.

-Eh bien, je vais marcher aujourd'hui...

(Niane, 1960, p.43)

Un tel épisode qui consacre le réveil $d u$ lion ne marque pas la fin des souffrances du héros qui sera obligé de s'exiler. C'est ce que Sellier résume en parlant de l'exposition. Même si dans ce cas précis il ne s'agit pas d'un nouveau-né comme dans les récits épiques classiques, cette phase s'observe toujours dans l'enfance du héros et suit l'occultation.

Pour accéder au grade de héros et d'immortel, il faut plusieurs naissances :

Les récits dans lesquels s'est exprimé le désir d'héroïsme, d'arrachement à la banalité de la vie, de supériorité sur le reste du monde, d'élévation à une condition quasi divine forment...l'épopée. L'analyse de ces productions conduit à discerner, sous les variations, un thème fondamental... : la manifestation de plus en plus éclatante du héros par des naissances successives jusqu'à sa naissance immortelle. La progression est rythmée par l'alternance naissance-mort-naissance (Sellier 1970)

La première mort symbolique de Soundjata s'est observée durant son exil. Sassoume la marâtre du héros réussit à l'écarter du pouvoir après la mort du Roi. La famille de Soundjata sera alors contrainte à quitter Niani la capitale manding pour s'installer dans un premier temps à Djedeba puis chez le Roi Fran Kamara à Tabon. Cet exil va marquer l'affirmation de son statut de héros par des signes multiples notamment la bravoure, l'endurance, la puissance. Soundjata sera initié au combat et deviendra très jeune Kan-koro-Sigui c'est-a-dire viceroi :

Au bout de trois ans, le Roi nomma Soundjata Kan-Koro-Sigui, c'est-à-dire vice-roi; en l'absence du Roi c'était lui qui commandait. Djata avait maintenant dixhuit hivernages. C'était alors un grand jeune homme au gros cou, à la poitrine puissante; personne ne pouvait tendre 
son arc.Tout le monde s'inclinait devant lui, on l'aimait; ceux qui nel'aimaient pas le craignaient; sa voix devint autoritaire. Le choix du Roi fut approuvé (Niane, 1960, p.71)

Le triomphe du héros qui confirme sa supériorité sur ses contemporains, sera rendu possible non seulement pas sa force physique mais surtout par ses qualités de fin stratège qui lui feront tisser des liens avec d'autres Rois avec qui il partage le même combat contre le cruel RoiSosso, Soumaoro Kanté. Ce dernier est l'incarnation des monstres (Minotaure, Sphinx ...) et des tirans (Goliath...) que combattaient les héros mythiques classiques (Hercule, Thésée, David...). C'est là où réside tout le mérite du héros selon Sellier (1970) c'est-à-dire réussir à réaliser ce qu'aucun être n'a pu faire :

Il se lance dans une de ces entreprises inouïes qui, à l'instar du combat contre le monstre, manifestent son être épiphanique ... Ces entreprises inouïes... dont aucun des mortels ordinaires n'est jamais revenu. Mais lui il en revient.

Niane revient largement sur la fin de l'exil au chapitre "Le retour »Il y relate le retour du héros au Manding, royaume de son défunt père, pour reconquérir le trône qui lui revient de droit. Il sort vainqueur de la bataille de Kirina qui se termine par la mort du tyrannique RoiSosso caricaturé dans l'œuvre comme un monstre au visage humain :

Comme tous les maîtres du feu, Soumaoro Kantéétait un grand sorcier ;la puissance de ses fétiches était terrible,c'était à cause de cesfétiches que tous les rois tremblaient devant lui, car il pouvait lancer la mort sur qui il voulait.Il avait fortifié Sosso....Il s'était fait construireune immense tour de sept étages et il habitaitau septième étage aumilieu de ses fétiches, c'est pourquoi on l'appelait le « roi intouchable » (Niane, 1960, p.79).

Cette victoire sur celui qui était considéré comme invincible, marque la fin des exploits du héros et son retour au niveau d'homme donc exposé à la perte de la vie comme fin naturelle de tout être. Selon
Djibril TamsirNiane, Soundjata serait emporté par les eaux de la rivière Sankara puis inhumer dans les rives de ce cours d'eau. Mais sa disparition demeure encore mystérieuse. Cette mort physique du héros trouve tout son sens, dans la réalisation $\mathrm{du}$ désir d'être dieu c'est-à-dire d'atteindre l'immortalité. L'apothéose dont parlait Sellier qui désigne un triomphe contre la mort, une immortalisation s'applique bien, ici.

À l'instar des héros épiques qui bénéficient d'un hymne à leur honneur pour magnifier leur grandeur et les ancrer durablement dans la postérité, Soundjata a aussi ses chansons qui le glorifient et l'éternisent.Ainsi, s'inspirant de Niane, Kesteloot (2010) nous rapporte ces vers :

Je chante, j'appelle ici Soundiata

Le fils de Sogolon, la femme-buffle

Le fils des Konaté-Keïta de la lignée des lions.

J'appelle le fondateur de l'empire du Mali.

Je chante celui qui a réuni dix royaumes Depuis le fond du Soudan jusqu'à l'océan Peuplés de gens parlant dix langues Qui n'arrêtaient pas de se faire la guerre. J'évoque l'histoire du valeureux guerrier Qui sut combattre, vaincre, puis pacifier Le plus vaste empire de l'Afrique.

Soudjata, à l'instar des hérosclassiques, est parti mais il est toujours présent car ses exploits : baobab déraciné, immense barre de fer tordu, multiples prouesses à la chasse, reconquête $\mathrm{du}$ trône royal, libération de son peuple, fondation de l'empire du Mali, établissement de la charte de Kouroukan Fouga, continuent d'être glorifiés par des musiciens du Mali, racontés par des griots manding et enseignés dans plusieurs établissements d'Afrique et d'ailleurs.

\section{CONCLUSION}

La naissance du héros épique, son enfance, son affirmation de même que son double triomphe tant des épreuves de la vie que de la mort comme éléments constituants le modèle héroïque classique tel qu'il est présenté par Philippe Sellier sont intégralement inscrits dans Soundjata ou l'épopée mandingue de Djibril TamsirNiane. Ce 
qui nous permet de dire que malgré la diversité des cultures et des trajectoires historiques et les différences spatiales entre l'occident et l'Afrique subsaharienne, les épopées royales ont, pour l'essentiel, plusieurs points en commun. D'où le caractère universel de ce genre oral qui n'est l'apanage d'aucun peuple. Chaque communauté a ses récits épiques calqués sur le modèle générique qui s'appuie sur une ossature bien déterminée qui en constitue la constante alors que les entités spatiotemporelles, les figures historiques y compris les événements qui ont marqué leur existence, demeurent des variables. Au demeurant, si l'épopée dynastique négroafricaine se retrouve bien dans le modèle héroïque de Sellier, il serait difficile voir impossible, à priori, d'affirmer que c'est le cas des autres types de récits épiques notamment corporatifs, religieux ou mythologiques claniques.

\section{REMERCIEMENTS}

Le fonctionnement du laboratoire études africaines de l'université Cheikh Anta Diop de Dakar, est rendu possible grâce à d'éminents chercheurs et plus particulièrement le Professeur feu Bassirou Dieng qui s'est éteint l'année dernière en laissant derrière lui une production d'une richesse incalculable et faisant la fierté de tous les passionnés de la littérature négroafricaine. C'est donc à tous ces grands esprits qui nous ont montré la voie à suivre pour mieux faire connaitre les œuvres des auteurs de l'Afrique subsaharienne, que J'adresse mes plus sincères remerciements.

\section{RÉFÉRENCES}

Camara L. (1978). Le Maitre de la parole. Paris : Plon.

Cissé Y.T. (1988). La grande geste du Mali. Des origines à la fondation de l'empire. Paris : Karthala.

Cissé Y. T. (1991). Soundjata, la gloire du Mali. Paris : Karthala.

Diabaté.M. (1973). Essai critique sur l'épopée mandingue (Thèse de $3^{\mathrm{e}}$ cycle). Université Panthéon-Sorbonne.

Dieng , B. (1997). Les épopées d'Afrique noire, Paris : Karthala.

Dieng, S. (2009). Sur les traces d'Elhadj Omar et la structure $d u$ mythe héroïque de PhilippeSellier, cours polycopié. Dakar: UCAD.

Dumézil G. (1949). L'Héritage indo-européen à Rome. Paris : Gallimard.

Kesteloot, L. (2010). Soundjata: L'enfant-lion. Paris: Casterman.

Ly-Tall, M. (1977). L'Empire du Mali. Paris: NEA.

Musset, A. (1937). Les Nuits. Paris: 1837.

Niane D. T.(1960), Soundjata ou l'épopée mandingue, Paris : Présence africaine.

Rank O. (1909), Le Mythe de la naissance du héros: Essai d'une interprétation psychanalytique du mythe, Paris : Payot.

Revel N. (1966), L'Épopée, https://www.universalis.fr/encycloped ie/epopee/.

Sellier Ph. (1970), La structure du mythe $d u$ héros ou le désir d'être dieu, Paris: Bordas.

Zumthor P. (1982), Introduction à la poésie orale : Paris : Seuil. 